\title{
Loop-Tree Duality for Multiloop Numerical Integration
}

\author{
Zeno Capatti, Valentin Hirschi®, Dario Kermanschah, and Ben Ruijl \\ ETH Zürich, Rämistrasse 101, 8092 Zürich, Switzerland
}

(Received 21 June 2019; published 11 October 2019)

\begin{abstract}
Loop-tree duality (LTD) offers a promising avenue to numerically integrate multiloop integrals directly in momentum space. It is well established at one loop, but there have been only sparse numerical results at two loops. We provide a formal derivation for a novel multiloop LTD expression and study its threshold singularity structure. We apply our findings numerically to a diverse set of up to four-loop finite topologies with kinematics for which no contour deformation is needed. We also lay down the ground work for constructing such a deformation. Our results serve as an important stepping stone towards a generalized and efficient numerical implementation of LTD, which is applicable to the computation of virtual corrections.
\end{abstract}

DOI: 10.1103/PhysRevLett.123.151602

Introduction.-Loop integrals are an essential component of higher-order corrections to collider cross sections. Analytic techniques have enjoyed a durable success in this matter, but it has become increasingly evident that a further breakthrough necessitates a radical change of perspective. Numerical approaches are a promising alternative and have already been extensively explored for Feynman amplitudes using sector decomposition (see e.g., [1-7]). More recently, direct integration of finite loop integrals in fourdimensional Minkowskian momentum space have been considered together with the necessary complex contour deformation for handling integrable threshold singularities [8-11]. In this Letter, we study the possibility of rewriting an $n$-loop integral as a sum of terms with $n$ additional on shell conditions by analytically integrating over loop energies using a residue theorem. The ensuing identity is called loop-tree duality [12] (LTD). LTD is appealing from a numerical standpoint for at least four reasons: (1) the $n$-loop integral dimensionality is fixed to $3 n$ irrespective of the topology considered, (2) integrable singularities can be shown to be confined to a bounded volume [13] and are absent when considering certain kinematical configurations, (3) momentum-space divergent integrals naturally lend themselves to be regularized with local UV and IR counterterms [14-23], or even (4) through a direct combination with the corresponding real-emission contributions in the case of physical amplitudes [24-26].

In this Letter we derive a novel multiloop LTD expression by iteratively applying the residue theorem, carefully keeping track of the propagation of Feynman's causal

Published by the American Physical Society under the terms of the Creative Commons Attribution 4.0 International license. Further distribution of this work must maintain attribution to the author(s) and the published article's title, journal citation, and DOI. Funded by SCOAP ${ }^{3}$. prescription. In Ref. [27] an alternative multiloop LTD expression is derived by using distributional identities between dual and Feynman propagators instead of applying the residue theorem. The main distinction is that their final expression has more on shell conditions than loops. In Ref. [28] an LTD expression was presented where an averaging procedure over all contour closures was considered, invoking the multidimensional residue theorem. However, we found that their expression does not hold beyond one loop (the mistake has been acknowledged by the authors of [28], whereupon they revised their proof and main result, following our method of iteratively applying the residue theorem).

We proceed to numerically apply our LTD construction to various scalar loop topologies ranging from one to four loops. In all cases, we find agreement to better than $1 \%$, thereby validating our procedure and the expected structure of integrable as well as cancelling singularities exhibited by each term of our LTD expression. We also determine the constraints on a contour deformation and construct deformation vectors satisfying them. A first preliminary result is given for a two-loop LTD integration using a contour deformation.

Loop-tree duality formalism.-We consider the following general expression for an $n$-loop integral

$I=\int \prod_{j=1}^{n} \frac{d^{4} k_{j}}{(2 \pi)^{4}} \frac{N}{\prod_{i \in \mathbf{e}} D_{i}}, \quad D_{i}=q_{i}^{2}-m_{i}^{2}+\mathrm{i} \delta$

where $\mathbf{e}$ is the set of indices labeling the edges of a Feynman diagram and the numerator $N$ is a regular function of the loop momenta. The Feynman propagator $1 / D_{i}$ depends on the four-momentum $q_{i} \equiv\left(q_{i}^{0}, \vec{q}_{i}\right)$, the mass $m_{i}$, and the positive causal prescription $\mathrm{i} \delta$. We consider nonraised Feynman propagators, each with two first order poles in $q_{i}^{0}$ located at $\sigma E_{i} \equiv \sigma \sqrt{\vec{q}_{i}^{2}+m_{i}^{2}-\mathrm{i} \delta}, \sigma \in\{ \pm 1\}$, 
where $+E_{i}$ lies in lower complex half-plane. Introducing the signature vector $\mathbf{s}_{i}=\left(s_{i 1}, \ldots, s_{\text {in }}\right), s_{i j} \in\{ \pm 1,0\}$, we write $q_{i}^{\mu}=\sum_{j=1}^{n} s_{i j} k_{j}^{\mu}+p_{i}^{\mu}$, where $p_{i}^{\mu}$ is a shift that depends on external momenta.

The integration over the momenta $k_{j}$ can be split up in an integration over a spatial part $\vec{k}_{j}$ and the energy $k_{j}^{0}$. We now derive our LTD formula by performing the energy integrations one after the other, following an arbitrary fixed order of the energy variables $\mathbf{k}^{\mathbf{0}}=\left(k_{1}^{0}, \ldots, k_{n}^{0}\right)$. We construct this iterative procedure by considering a contour for each energy integration variable along the real line, and closing on an arc in either the upper (with winding number $\left.\Gamma_{j}=+1\right)$ or the lower $\left(\Gamma_{j}=-1\right)$ complex half-plane. We assume the integral along the arc to vanish, such that the integral along the real line equals the sum of residues at poles located within the contour. The final expression obtained after iteratively performing each loop energy integration reads

$I=\int \prod_{j=1}^{n} \frac{d^{3} \vec{k}_{j}}{(2 \pi)^{3}} \Gamma_{j} \mathrm{i} \sum_{\substack{\mathbf{i} \in \mathcal{I} \\ \sigma \in\{+1,-1\}^{n}}} \operatorname{Res}_{\mathbf{k}_{\mathbf{i}}^{\sigma}}[f] \prod_{r=1}^{n} \Theta\left(\Gamma_{r} \widetilde{\Im}\left[k_{\mathbf{i}, r}^{\sigma}\right]\right)$,

where we introduce the set of ordered lists of edge indices $\mathcal{I}=\left\{\left(i_{1}, \ldots, i_{n}\right) \in \mathbf{e}^{n} \mid \operatorname{det}\left[\left(s_{i_{j} j}\right)_{1 \leq j \leq r}\right] \neq 0, \forall r \leq n\right\}$, which guarantees that for every iteration $j$, where we integrate out $k_{j}^{0}$, the propagator labeled by $i_{j}$ depends on $k_{j}^{0}$. Note that this set can contain several permutations of the same indices. Each element $\mathbf{i}$ of the set $\mathcal{I}$ therefore corresponds to the ordered list of propagator indices containing the poles (with energy signs as given by $\boldsymbol{\sigma}$ ) considered by the residue $\operatorname{Res}_{\mathbf{k}_{\mathrm{i}}^{\sigma}}$.

The residue of $f=N / \prod_{i \in \mathrm{e}} D_{i}$ is

$\operatorname{Res}_{\mathbf{k}_{\mathbf{i}}^{\sigma}}[f]=\left.\frac{1}{\operatorname{det} \mathbf{s}_{\mathbf{i}}} \frac{1}{\prod_{r=1}^{n} \sigma_{r}} \frac{1}{\prod_{i \in \mathbf{i}} 2 E_{i}} \frac{N}{\prod_{i \in \mathbf{e} \backslash \mathbf{i}} D_{i}}\right|_{\mathbf{k}^{0}=\mathbf{k}_{\mathbf{i}}^{\sigma}}$,

being evaluated at the pole locations implicitly defined through the solutions $\mathbf{k}^{\mathbf{0}}=\mathbf{k}_{\mathbf{i}}^{\sigma}$ to the following linear system

$$
\left(\begin{array}{c}
\sigma_{1} E_{i_{1}} \\
\vdots \\
\sigma_{n} E_{i_{n}}
\end{array}\right)=\left(\begin{array}{c}
\mathbf{s}_{i_{1}} \\
\vdots \\
\mathbf{s}_{i_{n}}
\end{array}\right)\left(\begin{array}{c}
k_{1}^{0} \\
\vdots \\
k_{n}^{0}
\end{array}\right)+\left(\begin{array}{c}
p_{i_{1}}^{0} \\
\vdots \\
p_{i_{n}}^{0}
\end{array}\right) \equiv \mathbf{s}_{\mathbf{i}} \cdot \mathbf{k}^{\mathbf{0}}+\mathbf{p}_{\mathbf{i}}^{\mathbf{0}},
$$

where the signature matrix $\mathbf{s}_{\mathbf{i}}$ is a totally unimodular matrix. Each residue contributes to the integral if the pole location is within all contours of energy integrations already performed, corresponding to the condition $\Gamma_{r} \Im\left[k_{\mathbf{i}, r}^{\sigma}\right]>0$, $\forall r \leq n$. The imaginary part of the poles in the energy variable $k_{r}^{0}$ is computed using Cramer's rule for the last row of the subsystem of (4) arising after every iteration. Its final expression is given by the following [Eq. (5) remains unchanged for the case of complex-valued external kinematics. As pointed out by the authors of Ref. [28], proper analytic continuation requires that the imaginary part of external momenta do not contribute in that equation. Our final expression given in Eq. (6) is therefore also valid for the case of complex-valued external kinematics]:

$$
\Im\left[k_{\mathbf{i}, r}^{\sigma}\right]=\frac{\operatorname{det}\left(\begin{array}{cc} 
& \sigma_{1} \Im\left[E_{i_{1}}\right] \\
\left(s_{i_{j_{1}} j_{2}}\right)_{\substack{1 \leq j_{1} \leq r \\
1 \leq j_{2}<r}} & \vdots \\
& \sigma_{r} \Im\left[E_{i_{r}}\right]
\end{array}\right)}{\operatorname{det}\left[\left(s_{i_{j} j}\right)_{1 \leq j \leq r}\right]},
$$

which explicitly shows how the imaginary parts of poles selected by previous iterations propagate to the imaginary part of the pole contributing at iteration $r$.

Equation (2) contains Heaviside functions $\Theta$ with complicated arguments. However, we have checked that for all topologies from one to six loops the Heaviside functions that do not identically evaluate to either 0 or 1 cancel pairwise. In fact, we find that for each loop momentum basis of the corresponding loop graph, only one combination of energy signs contributes to $I$ with a definite prefactor $(-i)^{n}$. We call this combination of signs the cut structure. Therefore, we conjecture that Eq. (1) can be written as

$$
I=(-\mathrm{i})^{n} \int \prod_{j=1}^{n} \frac{d^{3} \vec{k}_{j}}{(2 \pi)^{3}} \sum_{\mathbf{b} \in \mathcal{B}} \operatorname{Res}_{\mathbf{b}}[f]
$$

where $\mathbf{b}$ is the set of all edge indices labeling a loop momentum basis (a loop momentum basis is a set $\left\{q_{i}\right\}_{i \in \mathbf{b}}$ such that any other propagator momentum is expressed as a unique linear combination of the $\left\{q_{i}\right\}_{i \in \mathbf{b}}$ plus a translational term which only depends on external kinematics) and $\mathcal{B}$ is the set of these sets for all loop momentum bases. The cut structure of $\mathbf{b}$ is denoted with $\boldsymbol{\sigma}^{\mathbf{b}}$. Each loop momentum basis is assigned a residue, henceforth referred to as a dual integrand, reading

$$
\operatorname{Res}_{\mathbf{b}}[f]=\left.\frac{1}{\prod_{i \in \mathbf{b}} 2 E_{i}} \frac{N}{\prod_{i \in \mathbf{e} \backslash \mathbf{b}} D_{i}}\right|_{\left\{q_{j}^{0}=\sigma_{j}^{\mathbf{b}} E_{j}\right\}_{j \in \mathbf{b}}},
$$

where solving $\left\{q_{j}^{0}=\sigma_{j}^{\mathbf{b}} E_{j}\right\}_{j \in \mathbf{b}}$ yields $\mathbf{k}^{\mathbf{0}}=\mathbf{k}_{\mathbf{b}}^{\boldsymbol{\sigma}^{\mathbf{b}}}$. Note that the dual integrand is invariant under permutations of the elements in $\mathbf{b}$, unlike Eq. (3) that depends on the ordering within i. Furthermore, the complement $\mathbf{t}=\mathbf{e} \backslash \mathbf{b}$ is the spanning tree of the graph. There is a one-to-one correspondence between a spanning tree $\mathbf{t}$ and a loop momentum basis $\mathbf{b}$, hence the name loop-tree duality.

As an example, we consider the two-loop vacuum bubble with its three propagators with momenta $q_{1}=k_{1}$, $q_{2}=k_{1}+k_{2}$, and $q_{3}=k_{2}$. Then, when choosing to integrate first $k_{1}^{0}$ and then $k_{2}^{0}$ (both times opting to close 
our integration contour in the lower-half complex plane), we obtain one cut structure for each of the three possible momentum bases: $\boldsymbol{\sigma}^{\{1,2\}}=\{-1,+1\}, \boldsymbol{\sigma}^{\{1,3\}}=\{+1,+1\}$, and $\boldsymbol{\sigma}^{\{2,3\}}=\{+1,+1\}$. We provide a more detailed threeloop example in the Supplemental Material [29].

We expect the sum of residues obtained from analytic integration of loop energies to be independent of the specific loop momentum routing as well as choice of contour closure for each loop energy integration. We verified that these expectations are met by explicitly applying Eq. (6) for various choices of routing and contour closures, each time retrieving the same numerical result for the sum of residues $\sum_{\mathbf{b} \in \mathcal{B}} \operatorname{Res}_{\mathbf{b}}[f]$ for given numerical inputs $\vec{k}_{j}$. In performing these checks, it was convenient to have the cut structure construction algorithm automated and we provide the corresponding PYTHON implementation as Supplemental Material [29].

We stress that our procedure described in Eqs. (2)-(5) for obtaining the cut structure of an arbitrary topology has a negligible computational load. The interesting question of whether this cut structure can be obtained in a more direct fashion is therefore of theoretical interest only and beyond the scope of this Letter. Indeed, for all practical purposes one can consider using Eq. (6) directly as an alternative representation of the original integral.

Singular surfaces: Performing the energy integrations introduces additional dependencies on the regulator $\delta$ in the integrand $\sum_{\mathbf{b} \in \mathcal{B}} \operatorname{Res}_{\mathbf{b}}[f]$. For vanishing $\delta$, the dual propagator associated with the loop momentum basis $\mathbf{b}$ reads

$$
\frac{1}{\left.D_{i}\right|_{\left\{q_{j}^{0}=\sigma_{j}^{\mathbf{b}} E_{j}\right\}_{j \in \mathbf{b}}}}=\frac{1}{\left(\left.q_{i}^{0}\right|_{\left\{q_{j}^{0}=\sigma_{j}^{\mathbf{b}} E_{j}\right\}_{j \in \mathbf{b}}}\right)^{2}-\left(E_{i}\right)^{2}}
$$

and still features singularities if it can go on shell. The inverse dual propagator vanishes on two singular surfaces,

$$
S_{i}^{\mathbf{b}, \sigma}: \Delta_{i}^{\sigma, \mathbf{b}} \equiv p_{i}^{0, \mathbf{b}}+\sigma E_{i}+\sum_{j \in \mathbf{b}} s_{i j}^{\mathbf{b}} \sigma_{j}^{\mathbf{b}} E_{j}=0,
$$

where $\sigma \in\{ \pm 1\}$, and where $s_{i j}^{\mathbf{b}}$ and $p_{i}^{0, \mathbf{b}}$ are defined implicitly through the change of basis $q_{i}^{0}=$ $\sum_{j \in \mathbf{b}} s_{i j}^{\mathbf{b}} q_{j}^{0}+p_{i}^{0, \mathbf{b}}$.

The singular surfaces can be separated into two classes, which we call $E$ and $H$ surfaces. To distinguish them, we define the surface signs for the surface $S_{i}^{\mathbf{b}, \sigma}$ as the list $\mathcal{S}_{i}^{\mathbf{b}, \sigma}=\left\{s_{i j}^{\mathbf{b}} \sigma_{j}^{\mathbf{b}}, \quad \forall j \in \mathbf{b} \mid s_{i j}^{\mathbf{b}} \neq 0\right\} \cup\{\sigma\}$. A singular surface where all surface signs are equal is called an $E$ surface, since its defining equation is the one of an ellipsoid when $n-1$ loop momenta are kept fixed. Otherwise, it is called an $H$ surface, since its equation is the one of a hyperboloid when viewed as a function of at least one loop momentum.

We now provide the multiloop existence conditions for $H$ surfaces. In the one-loop case, and in general when $\left|\mathcal{S}_{i}^{\mathbf{b}, \sigma}\right|=2$, we have $\{j\} \equiv\left\{k \in \mathbf{b} \mid s_{i k}^{\mathbf{b}} \neq 0\right\}$ and the
$H$ surface exists for real masses and loop momenta if and only if

$$
\left(p_{i}^{0, \mathbf{b}}\right)^{2}-\vec{p}_{i}^{2}<\left(m_{j}-m_{i}\right)^{2},
$$

as already found in Ref. [30]. In the case of $\left|\mathcal{S}_{i}^{\mathbf{b}, \sigma}\right|>2$ and if exactly one $H$ surface sign differs from the others, whose index in $\mathbf{b} \cup\{i\}$ we label $\tilde{e}$, we define the following quantity:

$$
\Delta M_{i}=\sum_{j \in \mathbf{b}}\left|s_{i j}^{\mathbf{b}}\right|(-1)^{\delta_{\bar{e} j}} m_{j}+(-1)^{\delta_{\bar{e} i}} m_{i},
$$

and the corresponding $H$ surface exists if and only if

$\sigma_{\tilde{e}} p_{i}^{0, \mathbf{b}}<0$ and $\Delta M_{i}<0$ and $\left(p_{i}^{0, \mathbf{b}}\right)^{2}-\vec{p}_{i}^{2}<\left(\Delta M_{i}\right)^{2}$,

$\sigma_{\tilde{e}} p_{i}^{0, \mathbf{b}}>0$ and $\Delta M_{i}>0$ and $\left(p_{i}^{0, \mathbf{b}}\right)^{2}-\vec{p}_{i}^{2}>\left(\Delta M_{i}\right)^{2}$,

$\sigma_{\tilde{e}} p_{i}^{0, \mathbf{b}}>0$ and $\Delta M_{i}<0$,

or when the surface signs contain at least two positive and at least two negative members.

The singularities of dual integrands on $H$ surfaces cancel pairwise in their sum $\sum_{\mathbf{b} \in \mathcal{B}} \operatorname{Res}_{\mathbf{b}}[f]$, due to a mechanism referred to as dual cancellations [13,30,31], independently of the regulator $\delta$. We checked both numerically and analytically that Eq. (6) maintains the dual cancellation pattern of $H$ surfaces also beyond two loops.

$E$ surfaces satisfy the following existence conditions for real masses and loop momenta:

$\left(p_{i}^{0, \mathbf{b}}\right)^{2}-\vec{p}_{i}^{2} \geq\left(\sum_{j \in \mathbf{b}}\left|s_{i j}^{\mathbf{b}}\right| m_{j}+m_{i}\right)^{2}$ and $\sigma p_{i}^{0, \mathbf{b}}<0$.

We note that, when the bound above is saturated, the $E$ surface is said to be pinched and it corresponds to the location of physical soft and collinear singularities of the loop integral which would require dedicated local counterterms for its regularization.

The singularities on existing $E$ surfaces must be regularized through a contour deformation satisfying its corresponding $\delta$ prescription. We derive this prescription by writing the leading term of the Taylor expansion in $\delta$ of the imaginary part of $\Delta_{i}^{\sigma, \mathbf{b}}$ :

$$
\Im\left[\Delta_{i}^{\sigma, \mathbf{b}}\right]=-\frac{\delta}{2}\left(\frac{\sigma}{E_{i}}+\sum_{j \in \mathbf{b}} \frac{s_{i j}^{\mathbf{b}} \sigma_{j}^{\mathbf{b}}}{E_{j}}\right)+\mathcal{O}\left(\delta^{2}\right) .
$$

We note that for $E$ surfaces we have the definite sign $\operatorname{sgn} \Im\left[\Delta_{i}^{\sigma, \mathbf{b}}\right]=-\sigma$ independent of loop kinematics. If no $E$ surface existence condition is satisfied, the integrand $\sum_{\mathbf{b} \in \mathcal{B}} \operatorname{Res}_{\mathbf{b}}[f]$ has no singularities, and it is therefore independent of the regulator $\delta$. In this case, the numerical integration can be performed without a contour deformation, 
a feature that has already been shown at one loop in Ref. [30] and two loops in Ref. [32]. A first preliminary result for a two-loop LTD integration using a contour deformation will be given in the next section.

Numerical application. - LTD has shown to yield promising results at one loop [30] and has the advantage of not necessitating any computationally demanding symbolic treatment of the integrand and/or its numerator. This is different from sector decomposition techniques, which require building the Feynman representation of loop integrals together with the identification of sectors. Moreover, integration in momentum space is particularly appealing for its optimal scaling with the number of contributing scales. Compared to the 4D momentum space integration method described in Ref. [9], LTD has at least five advantages: (1) the dimension of the integration is reduced to three per loop, (2) a complex contour deformation only needs to be applied on bounded $E$ surfaces, (3) masses do not complicate the contour deformation much, (4) specific kinematical configurations can be integrated without any deformation, and (5) its singularity structure can directly be related to real-emission contributions [33].

In this Letter, we are mostly interested in demonstrating LTD viability for numerical multiloop computations and in assessing the validity of Eq. (6). Therefore, we apply LTD to loop integrals with external kinematics that do not yield singular $E$ surfaces, such that no complex contour deformation is required. This scenario offers a reliable numerical check of our LTD cut structures and of the numerical stability of the dual cancellations. Our implementation is a first important step towards handling loop integrals in the physical regime, which we briefly discuss in this section.

We selected eight very different loop topologies, displayed in Table II, to showcase the generality of the method. We report our results in Table I and Fig. 1, with additional information (such as the exact input kinematics) given as Supplemental Material [29] to ensure the reproducibility of our work. The reference results are taken from the analytic expression for the four-point integrals [34], from FORCER [35] for two-point integrals, from MADLOOP [36,37] for the decagon and triacontagon and PYSECDEC [7] for the six- and eight-point integrals (in which case the numerical error is also reported). We find perfect agreement in all cases, but note that scalar integrals whose superficial degree of UV divergence is -2 [Tables II(f) and II(h)] are numerically more challenging. This is made manifest for example when comparing LTD results obtained for the loops in Tables II(f) and II (g). We find no notable sensitivity of the numerical convergence to the external momenta multiplicity, internal masses, or nonplanarity of the loop graph.

For all eight benchmark loop integrals, we have explicitly verified that dual cancellations hold by sampling points on the $H$ surfaces for which we found that the sum of dual integrands is regular. It is important to monitor numerical
TABLE I. Comparison of our numerical LTD results for the topologies listed in Table II against either the analytic result [34,35] or an alternative numerical evaluation [7,36]. A star indicates that internal lines are set massive. The columns labeled $N$ and $[\mu \mathrm{s}]$ denote the Monte Carlo statistics and timing per sample respectively. See details (including kinematic configurations) in the Supplemental Material [29].

\begin{tabular}{llccll}
\hline \hline$G$ & & Reference & Numerical LTD & $N\left[10^{6}\right]$ & {$[\mu \mathrm{s}]$} \\
\hline a)* & {$[36]$} & $i 4.31638 \times 10^{-7}$ & $i 4.31637(19) \times 10^{-7}$ & 110 & 1.1 \\
b) & {$[36]$} & $i 0.358640$ & $i 0.358646(29)$ & 210 & 5.9 \\
c) & {$[7]$} & $1.1339(5) \times 10^{-4}$ & $1.133719(58) \times 10^{-4}$ & 5500 & 2.5 \\
c)* & {$[7]$} & $4.398(1) \times 10^{-8}$ & $4.39825(17) \times 10^{-8}$ & 5500 & 2.5 \\
d)* & {$[7]$} & $2.409(1) \times 10^{-8}$ & $2.40869(27) \times 10^{-8}$ & 5500 & 3.5 \\
e) & {$[34]$} & $-1.433521 \times 10^{-6}$ & $-1.4338(18) \times 10^{-6}$ & 1500 & 27.4 \\
f) & {$[35]$} & $\mathrm{i} 5.26647 \times 10^{-6}$ & $\mathrm{i} 5.236(38) \times 10^{-6}$ & 7000 & 3.3 \\
g)* & {$[7]$} & $\mathrm{i} 1.7790(6) \times 10^{-10}$ & $\mathrm{i} 1.77648(48) \times 10^{-10}$ & 22000 & 11 \\
$\mathrm{~h})$ & {$[35]$} & $-8.36515 \times 10^{-8}$ & $-8.309(31) \times 10^{-8}$ & 7000 & 15.8 \\
\hline \hline
\end{tabular}

stability when probing points close to such surfaces, as dual cancellations occur by cancelling large summands. We monitor this stability by testing the invariance of dual integrands under rotation of the spatial parts of the loop momenta integrated over. The more challenging loop integrals required a custom numerical stability rescue system that promotes the floating point arithmetic accuracy to quadruple precision when needed (which is about a factor of 30 slower). We note however that the introduction of a complex contour deformation mitigates the numerical severity of dual cancellations.

Since we are mostly interested in verifying our method at this stage, we stress that no effort was made to finetune the integrator, sample statistics or loop momenta parametrizations. Sizeable improvements can be expected from

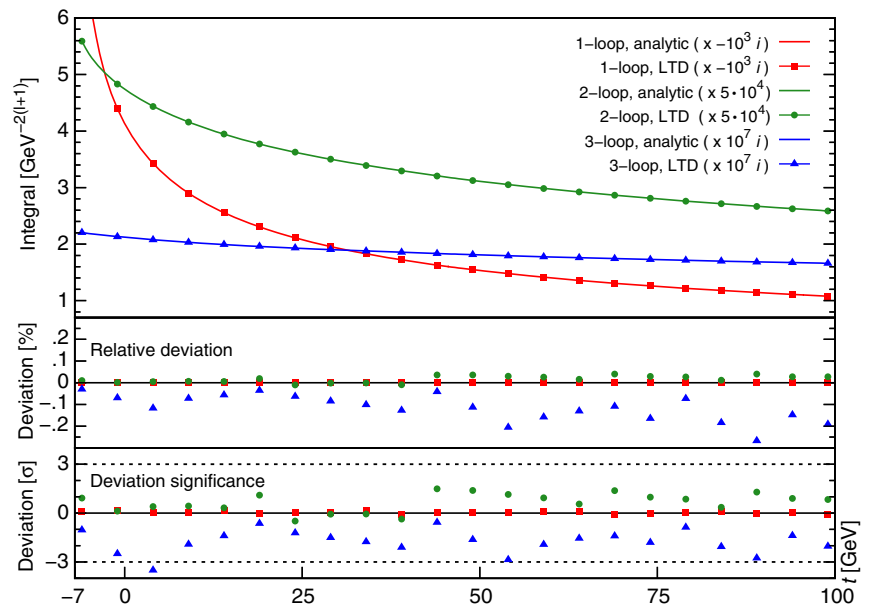

FIG. 1. Numerical LTD results obtained for the scalar massless one-, two-, and three-loop ladder box diagram with external kinematics satisfying (in $\mathrm{GeV}^{2}$ ) $p_{1}^{2}=-5, p_{i=2,3,4}^{2}=s=-1$ and values of the Mandelstam invariant $t$ ranging from $t=-7$ (loop threshold) to $t=100$. The analytic results are taken from Ref. [34]. 
TABLE II. Scalar loop diagrams considered in our numerical validation. A small line attached to a dotted vertex denotes an insertion of an external momentum. Graph (b) has 30 legs. (a)

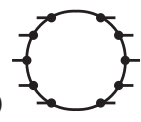

(b)

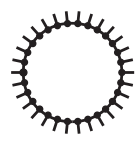

(c)

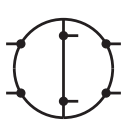

(d)

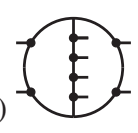

(e)

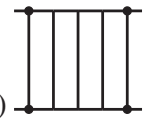

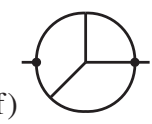

(g)

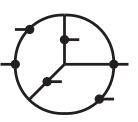

(h) considering techniques similar to the ones described in Ref. [16]. Similarly to what was found in Ref. [30], we observe that the CUHRE integrator offers significantly better convergence at one loop. However, we find that it performs much worse than VEGAS at higher loops. For uniformity, we restricted ourselves to using the VEGAS integrator for producing the results of Table I. Our implementation is written in the RUST language, with PYTHON bindings, and interfaces to the CUBA [38] library and VEGAS3.4 [39] for performing the adaptive Monte Carlo integration.

General kinematics.-The LTD expression of Eq. (6), evaluated at external kinematics relevant for computing physical scattering amplitudes, typically features singular $E$ surfaces. The corresponding singularities then require a complex contour deformation of the spatial part of the loop variables, constructed so as to satisfy the LTD prescription associated to the surface, presented expanded at the first order in $\delta$ in (14). In the first section, we found that the sign of the imaginary part of the defining equation of $E$ surfaces given in Eq. (9) reads:

$$
\operatorname{sgn} \Im\left[\Delta_{i}^{\mathbf{b}, \sigma}\right]=-\sigma
$$

We now aim at constructing a contour deformation that satisfies the causality constraints implied by the $i \delta$ prescription. Given its parametrization $\vec{k}_{l}^{\mathbb{C}}=\vec{k}_{l}+\mathrm{i} \vec{K}_{l}, \quad l \in$ $\{1, \ldots, n\}$, one has that $\vec{q}_{j} \rightarrow \vec{q}_{j}+\mathrm{i} \vec{\kappa}_{j}, \forall j \in \mathbf{b}$, and the imaginary part of every other propagator momentum can be expressed as a linear combination of $\left\{\vec{\kappa}_{j}\right\}_{j \in \mathbf{b}}$. This results in $\Delta_{i}^{\mathbf{b}, \sigma}$ acquiring an imaginary part,

$$
\Im\left[\Delta_{i}^{\mathbf{b}, \sigma}\right]=\sum_{j \in \mathbf{b}} s_{i j}^{\mathbf{b}} \vec{\kappa}_{j} \cdot\left(\frac{\sigma_{j} \vec{q}_{j}}{2 E_{j}}+\frac{\sigma \vec{q}_{i}}{2 E_{i}}\right)
$$

in the first order truncation of the expansion in $\sqrt{\sum_{j}\left|s_{i j}^{\mathbf{b}}\right| \vec{\kappa}_{j}^{2}}$. For $E$ surfaces, this simplifies to

$$
\Im\left[\Delta_{i}^{\mathbf{b}, \sigma}\right]=\frac{\sigma}{2} \sum_{j \in \mathbf{b}}\left|s_{i j}^{\mathbf{b}}\right| \vec{\kappa}_{j} \cdot \vec{v}_{i, j}^{\mathbf{b}}, \quad \vec{v}_{i, j}^{\mathbf{b}}=\frac{\vec{q}_{j}}{E_{j}}+\frac{s_{i j}^{\mathbf{b}} \vec{q}_{i}}{E_{i}},
$$

which can be matched with (15) on individual $E$ surfaces by just setting $\vec{\kappa}_{j} \propto-\vec{v}_{i, j}^{\mathbf{b}}, \quad \forall j \in \mathbf{b}$ such that $s_{i j}^{\mathbf{b}} \neq 0$. Now let $\mathrm{H}(w)=\left\{v \in \mathbb{R}^{3} \mid w \cdot v>0\right\}$. One can observe that for every value of the loop variables

$$
\vec{q}_{j} \in \bigcap_{l \in \mathcal{E}_{j}^{\mathbf{b}}} \mathrm{H}\left(\vec{v}_{l, j}^{\mathbf{b}}\right), \quad \forall j \in \mathbf{b},
$$

where $\mathcal{E}_{j}^{\mathbf{b}}=\left\{l \in \mathbf{e} \backslash \mathbf{b}|| s_{l j}^{\mathbf{b}} \mid \vec{v}_{l, j}^{\mathbf{b}} \neq \overrightarrow{0}\right\}$. Thus, $\vec{\kappa}_{j} \propto-\vec{q}_{j}, \forall j \in \mathbf{b}$ satisfies the prescription on arbitrarily many $E$ surfaces associated to the same loop momentum basis $\mathbf{b}$, including on their intersection. Indeed, $\vec{q}_{j}$ has positive projection on all nonzero $\left|s_{i j}\right| \vec{v}_{i, j}^{\mathbf{b}}$ which might appear as summands in (17). This fails only if there exists an $E$ surface $S_{l}^{\mathbf{b}, \sigma}$ such that $\left|s_{l j}\right| \vec{v}_{l, j}^{\mathbf{b}}=\overrightarrow{0}, \forall j \in \mathbf{b}$, which would correspond to a pinched surface necessitating a soft and/or collinear regulator or subtraction. Finally, the intersections of two surfaces $S_{a}^{\mathbf{b}, \sigma}$ and $S_{b}^{\tilde{\mathbf{b}}, \tilde{\sigma}}$ with $\mathbf{b} \neq \tilde{\mathbf{b}}$ and $S_{a}^{\mathbf{b}, \sigma} \nsubseteq S_{b}^{\tilde{\mathbf{b}}, \tilde{\sigma}}, S_{b}^{\tilde{\mathbf{b}}, \tilde{\sigma}} \nsubseteq S_{a}^{\mathbf{b}, \sigma}$ lie on dual cancelling surfaces. We stress that the above does not provide a complete recipe for building an overall continuous deformation direction $\vec{K}_{l}$ satisfying all causal constraints and common to all dual integrands so as to preserve dual cancellations. This requires a (numerically efficient) strategy for interpolating between the deformation directions identified in Eq. (18) for each group of $E$ surfaces. Additionally, special care must be taken when setting the normalization of the resulting deformation vector $\vec{K}_{l}$.

We conclude this section by presenting a first two-loop numerical result from applying LTD to a double-box topology that requires a deformation around its 13 distinct $E$ surfaces. We set the external kinematics identical to those of the benchmark point chosen in Ref. [9] and also report them in the Supplemental Material [29]. Using VEGAS3.4 with 105M Monte Carlo samples, we obtained $-5.877(55) \times 10^{-14}$, which stands within $1 \%$ of the analytical result $-5.8973 \times 10^{-14}$. We will provide a general and numerically efficient contour deformation for multiloop LTD in an upcoming publication.

Conclusion.-We derived a novel expression for multiloop LTD that involves taking as many on shell conditions as there are loops. We demonstrated its potential for numerical integration by applying it to eight finite scalar multiloop topologies. Additionally, we gave a first result of a contour deformation at two loops, showing that LTD can be used for computing integrals with physical kinematics as well.

Our future work concerns extending the application of LTD to diagrams and loop amplitudes featuring (1) complicated overlaps of $E$ surfaces requiring a general contour deformation, and (2) UV and IR divergences, by designing local subtraction counterterms that leverage known factorization properties, such as the ones introduced in Ref. [22]. 
We would like to thank Andrea Pelloni and Babis Anastasiou for useful discussions. This project has received funding from the European Research Council (ERC) under Grant Agreement No. 694712 (PertQCD) and SNSF Grant No. 179016. Numerical results presented in this Letter used computational resources from the Piz Daint cluster, administered by the Swiss National Supercomputing Centre (CSCS).

[1] K. Hepp, Commun. Math. Phys. 2, 301 (1966).

[2] M. Roth and A. Denner, Nucl. Phys. B479, 495 (1996).

[3] T. Binoth and G. Heinrich, Nucl. Phys. B585, 741 (2000).

[4] C. Anastasiou, K. Melnikov, and F. Petriello, J. High Energy Phys. 09 (2007) 014.

[5] G. Heinrich, Int. J. Mod. Phys. A 23, 1457 (2008).

[6] A. V. Smirnov, Comput. Phys. Commun. 204, 189 (2016).

[7] S. Borowka, G. Heinrich, S. Jahn, S. P. Jones, M. Kerner, J. Schlenk, and T. Zirke, Comput. Phys. Commun. 222, 313 (2018).

[8] W. Gong, Z. Nagy, and D. E. Soper, Phys. Rev. D 79, 033005 (2009).

[9] S. Becker and S. Weinzierl, Eur. Phys. J. C 73, 2321 (2013).

[10] S. Becker and S. Weinzierl, Phys. Rev. D 86, 074009 (2012).

[11] C. Anastasiou, S. Beerli, and A. Daleo, Phys. Rev. Lett. 100, 241806 (2008).

[12] S. Catani, T. Gleisberg, F. Krauss, G. Rodrigo, and J.-C. Winter, J. High Energy Phys. 09 (2008) 065.

[13] J. J. Aguilera-Verdugo, F. Driencourt-Mangin, J. Plenter, S. Ramírez-Uribe, G. Rodrigo, G. F. R. Sborlini, W. J. Torres Bobadilla, and S. Tracz, arXiv:1904.08389.

[14] S. Becker, C. Reuschle, and S. Weinzierl, J. High Energy Phys. 12 (2010) 013.

[15] S. Becker, D. Goetz, C. Reuschle, C. Schwan, and S. Weinzierl, Proc. Sci., RADCOR2011 (2011) 008 [arXiv: 1112.3521].

[16] S. Becker, C. Reuschle, and S. Weinzierl, J. High Energy Phys. 07 (2012) 090.

[17] S. Becker, D. Goetz, C. Reuschle, C. Schwan, and S. Weinzierl, Proc. Sci., LL2012 (2012) 039 [arXiv:1209.2846].
[18] G. F. R. Sborlini, F. Driencourt-Mangin, R. HernandezPinto, and G. Rodrigo, J. High Energy Phys. 08 (2016) 160.

[19] G. F. R. Sborlini, F. Driencourt-Mangin, and G. Rodrigo, J. High Energy Phys. 10 (2016) 162.

[20] S. Seth and S. Weinzierl, Phys. Rev. D 93, 114031 (2016).

[21] F. Driencourt-Mangin, G. Rodrigo, and G. F. R. Sborlini, Eur. Phys. J. C 78, 231 (2018).

[22] C. Anastasiou and G. Sterman, J. High Energy Phys. 07 (2019) 056.

[23] R. Baumeister, D. Mediger, J. Pečovnik, and S. Weinzierl, Phys. Rev. D 99, 096023 (2019).

[24] R. J. Hernandez-Pinto, G. F. R. Sborlini, and G. Rodrigo, J. High Energy Phys. 02 (2016) 044.

[25] B. Page and R. Pittau, Eur. Phys. J. C 79, 361 (2019).

[26] R. Runkel, Z. Szőr, J. P. Vesga, and S. Weinzierl, arXiv: 1906.02218 .

[27] I. Bierenbaum, S. Catani, P. Draggiotis, and G. Rodrigo, J. High Energy Phys. 10 (2010) 073.

[28] R. Runkel, Z. Szőr, J. P. Vesga, and S. Weinzierl, Phys. Rev. Lett. 122, 111603 (2019); , 123, 059902 (2019);

[29] See the Supplemental Material at http://link.aps.org/ supplemental/10.1103/PhysRevLett.123.151602 for a Python implementation of the work presented in this letter as well as a three-loop example.

[30] S. Buchta, G. Chachamis, P. Draggiotis, and G. Rodrigo, Eur. Phys. J. C 77, 274 (2017).

[31] S. Buchta, G. Chachamis, P. Draggiotis, I. Malamos, and G. Rodrigo, J. High Energy Phys. 11 (2014) 014.

[32] F. Driencourt-Mangin, G. Rodrigo, G. F. R. Sborlini, and W. J. Torres Bobadilla, J. High Energy Phys. 02 (2019) 143.

[33] R. J. Hernandez-Pinto, G. F. R. Sborlini, and G. Rodrigo, J. High Energy Phys. 02 (2016) 044.

[34] N. I. Usyukina and A. I. Davydychev, Phys. Lett. B 298, 363 (1993).

[35] B. Ruijl, T. Ueda, and J. A. M. Vermaseren, arXiv:1704 .06650 .

[36] V. Hirschi, R. Frederix, S. Frixione, M. V. Garzelli, F. Maltoni, and R. Pittau, J. High Energy Phys. 05 (2011) 044.

[37] J. Alwall, R. Frederix, S. Frixione, V. Hirschi, F. Maltoni, O. Mattelaer, H. S. Shao, T. Stelzer, P. Torrielli, and M. Zaro, J. High Energy Phys. 07 (2014) 079.

[38] T. Hahn, Comput. Phys. Commun. 168, 78 (2005).

[39] G. P. Lepage, J. Comput. Phys. 27, 192 (1978). 